\title{
Investigating a Reliable Covariance Control Scheme For MDOF Systems
}

\author{
Richard V. Field, Jr. \\ Structural Dynamics and Vibration Control Department \\ SAND- $-98-072 / C$ \\ Sandia National Laboratories \\ Albuquerque, NM 87185-0439 \\ rvfield@sandia.gov \\ CONF- $980623-$ \\ Lawrence A. Bergman \\ Department of Aeronautical and Astronautical Engineering \\ University of Illinois \\ Urbana, IL 61801 \\ lbergman@uiuc.edu

\section{AECEIVED} \\ MAR 251998 \\ OSTI
}

\section{Abstract}

The authors attempt to extend their previous efforts towards a reliable control scheme that guarantees a specified degree of reliability for civil engineering structures. Herein, a two degree-of-freedom system is examined. Covariance control techniques are explored to design a compensator that will provide optimal closed-loop performance, while satisfying a constraint on system reliability. It was found for the system under examination that a stable control does not exist that also meets the target reliability level. Alternate formulations continue to be investigated.

\section{Introduction}

Reliability plays an important role in the design of civil engineering structures, with nearly all modern design codes now incorporating some recognition of uncertainty. One motivation for actively controlling these structures is the prospect of specifying the structural reliability implicitly in the design process.

Spencer, et al. [14] employed the reliability function as an integral cost which was maximized, subject to performance constraints, to obtain a reliable design of the closed loop system. Field and Bergman [3,4] employed a covariance control formulation in conjunction with classical dynamic reliability theory [10] to design controllers guaranteed to achieve a specific closed-loop reliability level over the life of the system. This approach was successfully applied to structures of one and two degrees-of-freedom in $[3,4]$. However, it was observed that the modal formulation employed in that work was inefficient for large scale structures, and alternate methods were sought.

Herein, a dynamic reliability formulation suggested by Lutes and Tzuang [9] is employed, in which the output to be regulated is the sum of the modal responses of the structure. While circumventing the inefficiencies associated with the previous modal formulation, this approach requires the ability to design to a specified output covariance; the mechanics of this are not as straightforward as those of the state covariance control formulation employed previously.

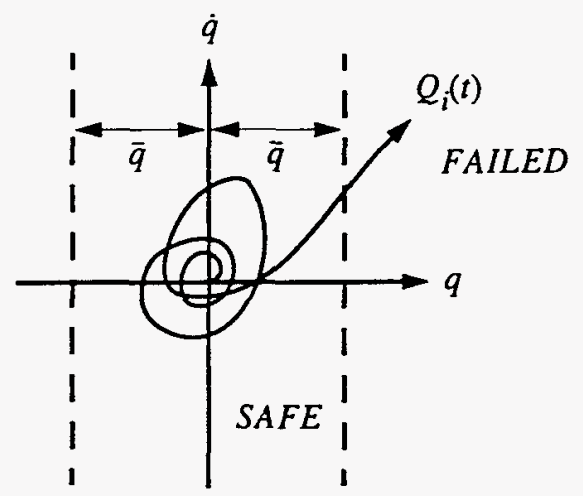

Fig. 1: One realization of $Q(t)$ outcrossing the safe domain.

Difficulties experienced with the formulation and solution of the problem are reported.

\section{Background}

Herein, two concepts are studied in detail: dynamic reliability and covariance control. Previously studied independent of one another, their interrelationship forms the foundation of this effort. A brief discussion of both is included for completeness.

\subsection{Dynamic Reliability}

Assessment of the reliability of a dynamic system requires the determination of the probability that the stochastic process representing system response, $Q(t)$, here assumed to be Gaussian and stationary, will cross out of a safe region into a failure region for the first time during a finite time interval, $t \in[0, T]$. This notion of failure can be expressed as

$$
p_{f}=1-P(|Q(t)| \leq \bar{q} ; t \in[0, T]),
$$

and is illustrated graphically in Fig. 1 for one sample function of the stochastic response process $Q(t)$.

Assuming that the probability of failure is small, it can be conservatively estimated in terms of the mean rate, $v_{D}$, at which $Q(t)$ outcrosses the boundary $\bar{q}$ into the failure region 


\section{DISCLAIMER}

This report was prepared as an account of work sponsored by an agency of the United States Government. Neither the United States Government nor any agency thereof, nor any of their employees, makes any warranty, express or implied, or assumes any legal liability or responsibility for the accuracy, completeness, or usefulness of any information, apparatus, product, or process disclosed, or represents that its use would not infringe privately owned rights. Reference herein to any specific commercial product, process, or service by trade name, trademark, manufacturer, or otherwise does not necessarily constitute or imply its endorsement, recommendation, or favoring by the United States Government or any agency thereof. The views and opinions of authors expressed herein do not necessarily state or reflect those of the United States Government or any agency thereof. 
[2]. A first-order approximation to the probability of system failure can then given by [13]

$$
p_{f}=1-e^{-v_{D} T}
$$

When considering single degree-of-freedom systems, the classical result of Rice [10] for the outcrossing rate can be used. This methodology, coupled with the reliability model introduced by Veneziano et al. [15], was applied in previous efforts by the authors in the area of reliable control for civil engineering structures $[3,4]$. This technique, however, did not lend itself to an efficient means of evaluating MDOF systems. Herein, the authors consider a different reliability model; namely, the model presented by Lutes and Tzuang [9], where system reliability is characterized by the sum of the system's modal response.

As discussed in [9], let

$$
Q(t)=\sum_{i} \eta_{i}(t)
$$

where each $\eta_{i}(t)$ is the response of a single degree-offreedom mode with damping $\zeta_{i}$ and natural frequency $\omega_{i}$. The excitations of the modes are perfectly correlated white noise processes, but generally exhibit different intensities. It is the sum of the modal responses, $Q(t)$, and its time derivative that are used to calculate the reliability of the system. The outcrossing rate for any level $d=a \sigma_{Q}$ for a normal process can be shown to be [9]

$$
v_{d}=\frac{1}{2 \pi} \frac{\sigma_{Q}}{\sigma_{Q}} \exp \left(\frac{-a^{2}}{2}\right),
$$

and an estimate of system reliability follows from Eq. (2).

\subsection{Covariance Control Theory}

The state covariance matrix contains vital information about closed-loop performance and stability. This motivates the following covariance control problem: find all controllers which assign a given state covariance to the closed-loop system. This given target state covariance will guarantee certain closed-loop performance specifications.

The notion of a covariance control theory was first introduced by Hotz and Skelton [6]. Since then, the concept has evolved into a control design scheme which addresses many modern control problems including those involving state feedback, static and dynamic output feedback, measurement noise, and minimum energy for both continuous- and discrete-time systems. A complete discussion of the covariance control theory is contained in [12].

For the problem formulation contained herein, covariance control as applied to the static output feedback problem is relevant. Consider the stable, actively controlled, linear time-invariant system shown in Fig. 2. When subjected to

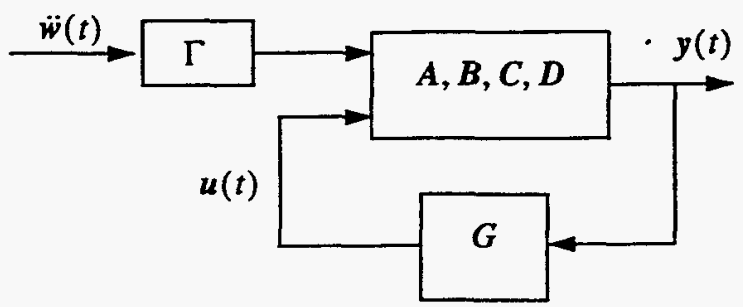

Fig. 2: Closed-loop system assuming static output feedback.

additive Gaussian white noise excitation, the equations of motion are

$$
\begin{gathered}
\underline{\dot{\eta}}(t)=\mathbf{A} \underline{\eta}(t)+\mathbf{B} u(t)+\Gamma \ddot{w}(t), \\
y(t)=\mathbf{C} \underline{\eta}(t), u(t)=\mathbf{G} y(t)
\end{gathered}
$$

$$
\begin{gathered}
\mathrm{A} \in \Re^{n_{p} \times n_{p}}, \mathrm{~B} \in \Re^{n_{p} \times n_{*}}, \mathrm{C} \in \Re^{n_{y} \times n_{p}}, \mathrm{G} \in \Re^{n_{u} \times n_{y}} \\
E[\ddot{w}(t)]=0, E\left[\ddot{w}(t) \ddot{w}^{T}(\tau)\right]=\mathrm{W} \delta(t-\tau), \mathbf{W}>0 .
\end{gathered}
$$

Here, $\underline{\eta}(t)$ is the $n_{p}$-dimensional state vector, written in terms of the modal coordinates.

It is of interest to find the set of all output feedback gains, $\mathbf{G}$, such that the closed-loop state covariance matrix, defined to be

$$
\mathbf{H} \equiv \lim _{\substack{t \rightarrow \infty \\ \tau \rightarrow 0}} E\left[\underline{\eta}(t) \underline{\eta}^{T}(\tau)\right],
$$

reaches some specified (positive definite) value, $\overline{\mathbf{H}}$. In the literature $[6,11,12]$, this is referred to as the State Covariance Assignment (SCA) problem.

Skelton et al. $[6,11,12]$ provide an overview of the SCA problem, which is briefly summarized here. When considering the static output feedback problem, the following theorem provides necessary and sufficient conditions to determine whether a specific $\overline{\mathbf{H}}$ is contained in $\Sigma$ (see [12] for proof).

Theorem: The static output feedback SCA problem may be assigned state covariance matrix $\overline{\mathbf{H}} \Leftrightarrow$

$$
\begin{gathered}
\left(\mathrm{I}-\mathbf{B B}^{+}\right)\left(\Gamma \mathbf{W} \Gamma^{T}+\mathbf{A} \overline{\mathbf{H}}+\overline{\mathbf{H}} \mathbf{A}^{T}\right)\left(\mathbf{I}-\mathbf{B B}^{+}\right)=\mathbf{0} \\
\left(\mathbf{I}-\mathbf{C}^{+} \mathbf{C}\right) \overline{\mathbf{H}}^{-1}\left(\Gamma \mathrm{W} \Gamma^{T}+\mathbf{A} \overline{\mathbf{H}}+\overline{\mathbf{H}} \mathbf{A}^{T}\right) \overline{\mathbf{H}}^{-1}\left(\mathbf{I}-\mathbf{C}^{+} \mathbf{C}\right)=\mathbf{0} \\
\left(\mathbf{I}-\mathbf{R} \mathbf{R}^{+}\right)\left(\mathbf{I}-\mathbf{C}^{+} \mathbf{C}\right) \overline{\mathbf{H}}^{-1} \mathbf{P}=\mathbf{0} \\
\text { where } \mathbf{R}=\left(\mathbf{I}-\mathbf{C}^{+} \mathbf{C}\right) \overline{\mathbf{H}}^{-1} \mathbf{B} \mathbf{B}^{+}
\end{gathered}
$$


Moreover, if the state covariance matrix satisfies these conditions, the set of all state feedback gain matrices, $\mathbf{G}$, that guarantee $\overline{\mathbf{H}}$ can be found from (see [12] for proof)

$$
\begin{gathered}
\{\mathbf{G}\}=\frac{1}{2} \mathbf{B}^{+}(\mathbf{P}+\mathbf{S}) \overline{\mathbf{H}}^{-1} \mathbf{C}^{+}, \\
\text {where } \mathbf{P}=\Gamma \Gamma^{T}+\mathbf{A} \overline{\mathbf{H}}+\overline{\mathbf{H}} \mathbf{A}^{T}, \\
\mathbf{S}=\left[\Theta^{+} \Psi+\left(\mathbf{I}-\Theta^{+} \Theta\right) S_{f}\right]\left(\mathbf{I}-\Theta^{+} \Theta\right)-\left(\Theta^{+} \Psi\right)^{T}, \\
\Theta=\left[\begin{array}{c}
\mathbf{I}-\mathbf{B} \mathbf{B}^{+} \\
\left(\mathbf{I}-\mathbf{C}^{+} \mathbf{C}\right) \overline{\mathbf{H}}^{-1}
\end{array}\right], \Psi=\left[\begin{array}{c}
-\left(\mathbf{I}-\mathbf{B} \mathbf{B}^{+}\right) \\
\left(\mathbf{I}-\mathbf{C}^{+} \mathbf{C}\right) \overline{\mathbf{H}}^{-1}
\end{array}\right] \mathbf{P} .
\end{gathered}
$$

Here, $S_{f}$ is arbitrary skew-symmetric and all $\mathbf{G} \in\{\mathbf{G}\}$ are stabilizing. In summary, this set of control gains ensures that the open-loop system of Eq. (5) will attain, in the steady-state, the target closed-loop covariance, $\overline{\mathbf{H}}$, provided the conditions of Eqs. (7) are met.

One final comment on the practical use of covariance control for output feedback problems. It has been discussed in $[8,11]$ that upper bounds on performance might be more useful than the more difficult task of assigning exact performance. This motivates the concept of the covariance upper bound control problem: determine if there exists a controller which stabilizes the system and yields an output covariance bounded above by a given matrix. As will be shown in the next section, it is difficult in practice to determine an admissible target covariance matrix for the covariance assignment problem. As a result, future work will investigate the possibilities of relating the dynamic reliability formulation of $\$ 2.1$ to the covariance upper bound control problem.

It remains to show how the concepts of dynamic reliability and covariance control complement one another. It can be shown, however, that this relationship is obvious, since a convenient way to choose a performance requirement is to place a hard constraint on the reliability of the system. This, in turn, leads to an objective for control design.

\section{Two Degree-of-Freedom Example}

The two degree-of-freedom system shown in Fig. 3 was utilized to illustrate the reliable control method. Assuming some type of actuation on the first cart, the dynamics of the controlled system are given by

$$
\left[\begin{array}{cc}
m & 0 \\
0 & m
\end{array}\right]\left[\begin{array}{l}
\ddot{x}_{1}(t) \\
\ddot{x}_{2}(t)
\end{array}\right]+\left[\begin{array}{cc}
2 k & -k \\
-k & k
\end{array}\right]\left[\begin{array}{l}
x_{1}(t) \\
x_{2}(t)
\end{array}\right]=\left[\begin{array}{l}
-m \\
-m
\end{array}\right] \ddot{w}(t)+\left[\begin{array}{l}
1 \\
0
\end{array}\right] u(t),
$$

where the $x_{i}$ 's are relative displacements (i.e., $x_{i}=z_{i}-w$ ), $m$ is the mass of each cart, $k$ is the stiffness of each spring, and $\ddot{w}(t)$ is the ground acceleration, modeled as a Gaussian white noise process. To proceed, convert to the modal space and apply modal damping

$$
\begin{gathered}
\mathrm{I} \ddot{\eta}(t)+\mathrm{Z} \dot{\eta}(t)+\Lambda \eta(t)=\Phi^{T} \gamma \ddot{\psi}(t)+\Phi^{T} \mathbf{b} u(t), \text { where } \\
\mathbf{Z}=\left[\begin{array}{cc}
2 \zeta_{1} \omega_{1} & 0 \\
0 & 2 \zeta_{2} \omega_{2}
\end{array}\right], \Lambda=\left[\begin{array}{cc}
\omega_{1}^{2} & 0 \\
0 & \omega_{2}^{2}
\end{array}\right], \gamma=\left[\begin{array}{l}
-m \\
-m
\end{array}\right], \\
\mathbf{b}=\left[\begin{array}{l}
1 \\
0
\end{array}\right] \text {, and } x(t)=\Phi \eta(t) .
\end{gathered}
$$

\subsection{Covariance Control Issues}

For covariance control purposes, Eq. (10) must be transformed to the state space

$$
\begin{gathered}
\underline{\eta}(t)=\mathbf{A} \underline{\eta}(t)+\mathbf{B} u(t)+\Gamma \ddot{x}(t) \text {, where } \mathbf{A}=\left[\begin{array}{cc}
0 & \mathbf{I} \\
-\Lambda & -\mathbf{Z}
\end{array}\right], \\
\mathbf{B}=\left[\begin{array}{c}
0 \\
\Phi^{T} \mathbf{b}
\end{array}\right], \Gamma=\left[\begin{array}{c}
0 \\
\Phi^{T} \gamma
\end{array}\right] \text {, and } \underline{\eta}(t)=\left[\begin{array}{c}
\eta(t) \\
\dot{\eta}(t)
\end{array}\right] .
\end{gathered}
$$

With the following output equation,

$$
y(t)=\mathbf{C} \underline{(t)}, \text { where } \mathbf{C}=\left[\begin{array}{llll}
1 & 1 & 0 & 0 \\
0 & 0 & 1 & 1
\end{array}\right] \text {, }
$$

the output vector is consistent with the reliability expressions of Eqs. (3) and (4)

$$
y(t)=Q(t)=\left[\begin{array}{c}
Q(t) \\
\dot{Q}(t)
\end{array}\right]=\left[\begin{array}{c}
\eta_{1}(t)+\eta_{2}(t) \\
\dot{\eta}_{1}(t)+\dot{\eta}_{2}(t)
\end{array}\right]
$$

With these preliminaries complete, the control design problem becomes: what static output feedback control will guarantee that the output vector attains a given target covariance? With this in mind, $\$ 3.2$ describes how to derive this target covariance matrix to assure the closed-loop system will attain a certain level of reliability.

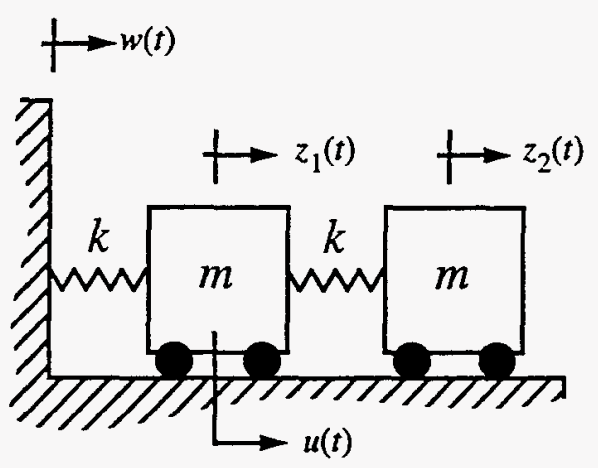

Fig. 3: Example two degree-of-freedom system. 


\subsection{Reliability Issues}

To address the reliability of the system, consider the following

$$
\text { Goal: }\left[\begin{array}{l}
\left|x_{1}\right| \\
\left|x_{2}\right|
\end{array}\right] \leq\left[\begin{array}{l}
d_{1} \\
d_{2}
\end{array}\right] \text { for } t \in[0, T] \text { with probability } p_{s} \text {. }
$$

This can also be stated in terms of the probability that the system will fail to meet the goal (i.e., the complement of $p_{s}$ )

$$
p_{f}=1-P(|X(t)| \leq d ; t \in[0, T])
$$

or equivalently,

$$
p_{f}=1-P(|Q(t)| \leq \bar{q} ; t \in[0, T])
$$

where

$$
\bar{q}=\left[\begin{array}{ll}
1 & 1
\end{array}\right] \Phi^{-1} d
$$

Assuming the failure probability, the time increment, and the safety level are all prescribed, a set of closed-loop variances exists, each of which guarantees the required degree of reliability of the system. This set follows directly from Eq. (4)

$$
\{\mathcal{V}\}=\left\{\left(\sigma_{Q}^{2}, \sigma_{\dot{Q}}^{2}\right): \frac{\sigma_{\dot{Q}}^{2}}{\sigma_{Q}^{2}}=\left[\frac{-2 \pi}{T} \ln \left(1-p_{f}\right) e^{\frac{\bar{q}^{2}}{2 \sigma_{Q}^{2}}}\right]^{2}\right\}
$$

To apply covariance control techniques, one must choose a pair from $\{\mathcal{V}\}$ (covariance of the output vector) that will cause the corresponding state covariance to satisfy the necessary conditions given in Eqs. (7). Therefore, knowledge of the relationship between state and output covariance is needed. Simple manipulation of the output equation gives the desired result

$$
E\left[y(t) y^{T}(\tau)\right]=E\left[\mathbf{C} \underline{\eta}(t) \underline{\eta}^{T}(\tau) C\right]=\mathbf{C} \overline{\mathrm{H}} \mathbf{C} .
$$

For this specific example,

$$
\mathbf{C} \overline{\mathbf{H}} \mathbf{C}=\left[\begin{array}{l}
\bar{h}_{11}+\bar{h}_{21}+\bar{h}_{12}+\bar{h}_{22} \bar{h}_{13}+\bar{h}_{23}+\bar{h}_{14}+\bar{h}_{24} \\
\bar{h}_{31}+\bar{h}_{41}+\bar{h}_{32}+\bar{h}_{42} \bar{h}_{33}+\bar{h}_{43}+\bar{h}_{34}+\bar{h}_{44}
\end{array}\right],
$$

where the $\bar{h}_{i j}$ are the individual element of $\overline{\mathbf{H}}$. Furthermore, $\overline{\mathbf{H}}$ is symmetric, so Eq. (20) reduces to three independent linear equations

$$
\begin{gathered}
\bar{h}_{11}+2 \bar{h}_{12}+\bar{h}_{22}=\sigma_{Q}^{2}, \\
\bar{h}_{33}+2 \bar{h}_{34}+\bar{h}_{44}=\sigma_{\dot{Q}}^{2}, \text { and } \\
\bar{h}_{13}+\bar{h}_{23}+\bar{h}_{14}+\bar{h}_{24}=0 .
\end{gathered}
$$

Because it represents the covariance of a physical system, elements of $\overrightarrow{\mathbf{H}}$ exhibit special properties

$$
\begin{gathered}
\bar{h}_{i i} \geq 0 \text { for all } i, \text { and } \\
\bar{h}_{12}, \bar{h}_{34}>0,
\end{gathered}
$$

where the first comes from the symmetry of $\overline{\mathbf{H}}$, and the last two conditions follow from observations in [9].

Choosing a target $\overline{\mathbf{H}}$ that satisfies the imposed reliability constraints and is admissible is no easy task. This is due to the fact that the necessary conditions of Eqs. (7) prove to be very stringent requirements. Regardless, a scheme to choose one such $\overline{\mathbf{H}}$ is given by

Solution scheme: Find all $\left(\bar{h}_{i j}, \sigma_{Q}, \sigma_{\dot{Q}}\right)$ such that

$\overline{\mathbf{H}}$ satisfies Eqs. (7) and Eqs. (21),

(iii) $\quad \bar{h}_{i j}$ satisfy Eqs. (22).

To solve this system of nonlinear equations, routines from MATLAB's Optimization Toolbox [5] were used. In particular FSOLVE with the Gauss-Newton algorithm was applied. However, after careful study of the two degree-offreedom problem using the scheme described above, a feasible solution was extremely difficult or impossible to achieve. The numerical scaling of Eq. (18) is poor when considering highly reliable systems, leading to an ill-conditioned problem. As a result, a solution was attained only when the reliability requirements were sufficiently relaxed (i.e., larger $p_{f}$ ). These results proved useless, however, since the reliability model of Eq. (2) is accurate only for small failure probabilities.

\section{Observations and Conclusions}

The application of covariance control methods can be advantageous when the control objective is to define the behavior of the state variables in the mean square. Previous work [4] illustrated how the theory of covariance control could successfully be applied to guarantee the reliability of a single degree-of-freedom system. Reliable control schemes for a three degree-of-freedom system with state feedback were introduced in [3].

Herein, attempts to derive a reliable control law for a two degree-of-freedom system with output feedback met with considerable hardship. After careful study, it was determined that the solution method described is successful only when the 
reliability constraints were sufficiently relaxed. As a result, another method to solve this problem must be derived if this work is to proceed.

One possible solution is to apply the covariance upper bound control schemes introduced in $[8,12]$. Instead of prescribing specific closed-loop performance, an upper bound can be utilized. As a result, the admissibility conditions on the state covariance matrix are relaxed and a feasible solution to this problem may be attained. To proceed in this manner, however, the map between system reliability and an upper bound on the response covariance must be attained.

\section{Acknowledgments}

The first authors' work was supported by the United States Department of Energy under Contract DE-AC04$94 \mathrm{AL} 85000$. Sandia is a multiprogram laboratory operated by Sandia Corporation, a Lockheed Martin Company, for the United States Department of Energy.

\section{References}

1. Belyaev, Y.K., "On the Number of Exits Across the Boundary of a Region by a Vector Stochastic Process," Theon of Probability Applications, 13, 1968, pp. 320324.

2. Ditlevsen, O., "Gaussian Outcrossings From Safe Convex Polyhedrons," ASCE Journal of Engineering Mechanics, 109(1), 1983, pp. 127-148.

3. Field, R.V. Jr., and L.A. Bergman, "A ReliabilityBased Approach to Linear Covariance Control Design," ASCE Journal of Engineering Mechanics, 124(2), pp. 193-199.

4. Field, R.V. Jr., and L.A. Bergman, "Reliability-Based Covariance Control Design," Proceedings of the American Control Conference, Albuquerque, NM, June, 1997, pp. 11-15. Also presented at the ASCE/ASMEJ SES McNU Symposium, July, 1997, Evanston, IL.

5. Grace, A., MATLAB ${ }^{\circledR}$ Optimization Toolbox, The MathWorks, Inc., Natick, MD, 1995.

6. Hotz, A. and R.E. Skelton, "A Covariance Control Theory," Proceedings of the 24th Conference on Decision and Control, Ft. Lauderdale, FL, December, 1985, pp. 552-557.

7. Kaspari, D.C. Jr., B.F. Spencer, Jr. and M.K. Sain, "Optimal Structural Control: A Reliability-Based Approach," Proceedings of the 1995 ASME Design Engineering Technical Conferences, 15th Biennial Conference on Mechanical Vibration and Noise, ASME DE-8401, pp. 855-862.

8. Lu, J. and R.E. Skelton, "Covariance Control Using Closed Loop Modeling for Structures," Proceedings of the 1997 ASCE Structures Congress, Portland, OR, 1997.

9. Lutes, L.D. and S. Tzuang, "First-Passage Probability for Two-Mode Systems," ASCE Journal of Engineering Mechanics, 109(6), pp. 1358-1374.
10. Rice, S.O., "Mathematical Analysis of Random Noise," Bell System Technical Journal, 23(282), 24(46), 1944. Also in Selected Papers on Noise and Stochastic Processes (N. Wax, ed.), Dover, New York, 1954.

11. Skelton, R.E. and M. Ikeda, "Covariance Controllers for Linear Continuous-Time Systems," International Journal of Control, 49(5), 1989, pp. 1773-1785.

12. Skelton, R.E., K. Grigoriadis, and T. Iwasaki, Control Systems Design: A Unified Algebraic Approach, notes from Workshop Number 5, American Control Conference, Albuquerque, NM, 1997.

13. Soong, T.T. and M. Grigoriu, Random Vibration of Mechanical and Structural Systems, PTR PrenticeHall, Inc., Englewood Cliffs, NJ, 1993.

14. Spencer, B.F. Jr., D.C. Kaspari, Jr., and M.K. Sain, "Structural Control Design: A Reliability-Based Approach," Proceedings of the American Control Conference, Baltimore, MD, June, 1994, pp. 10621066.

15. Veneziano, D., M. Grigoriu, and C. Allin Cornell, "Vector-Process Models for System Reliability," ASCE Journal of Engineering Mechanics, 1977, pp. 441-460.

\section{Nomenclature}

A, B, C, $\Gamma \quad$ equations of motion in state-space

G

$\overline{\mathbf{H}}$ output feedback gain matrix

$Q(t)$

$Q_{i}(t)$

$T, a, d$

$k, m$

$p_{f}, p_{s}$

$q, \dot{q}$

$\ddot{w}(t)$

$x(t)$

$\mathbf{Z}, \Lambda$

$\Phi$

$\eta_{i}(t)$

$\eta(t)$

$\underline{\eta}(t)$

$\sigma_{Q}^{2}, \sigma_{\dot{Q}}^{2}$ target state covariance matrix

vector response process with $Q(t)=$ transpose $[Q(t) \dot{Q}(t)]$ deterministic realization of $Q(t)$ reliability specifications stiffness and mass coefficients probability of failure and stability deterministic realizations of $Q$ and $\dot{Q}$

white noise excitation process with intensity $W$

response vector process with $x(t)=\operatorname{transpose}\left[\begin{array}{ll}x_{1}(t) & \left.x_{2}(t)\right]\end{array}\right.$ modal damping and stiffness

modal matrix

scalar response of mode $i$ vector modal response with $\eta(t)=$ transpose $\left[\eta_{1}(t) \eta_{2}(t)\right]$ state vector in modal coordinates variances of position and velocity response processes, respectively 


\section{M98004237}

|||||||||||||||||||||||||||||||||||||||||||||||||||||||||||||||

Report Number (14)SAN D- $-98-0721 \mathrm{C}$ conf-980623--

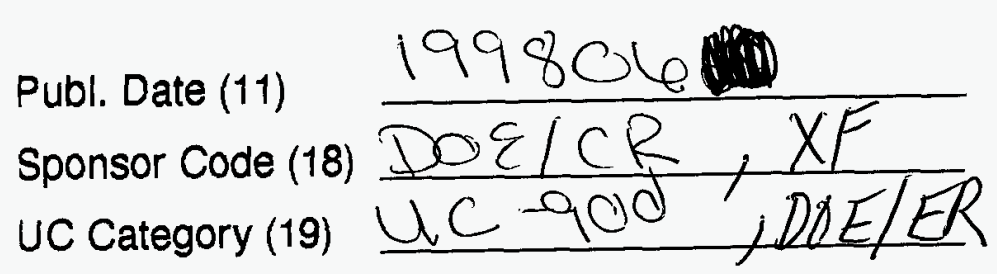

